\title{
Design of a Lightweight Soft Robotic Arm Using Pneumatic Artificial Muscles and Inflatable Sleeves
}

\author{
Preston Ohta,' Luis Valle, Jonathan King,, Kevin Low, Jaehyun Yi,,4 \\ Christopher G. Atkeson, and Yong-Lae Park ${ }^{1,3,4}$
}

\begin{abstract}
As robots begin to interact with humans and operate in human environments, safety becomes a major concern. Conventional robots, although reliable and consistent, can cause injury to anyone within its range of motion. Soft robotics, wherein systems are made to be soft and mechanically compliant, are thus a promising alternative due to their lightweight nature and ability to cushion impacts, but current designs often sacrifice accuracy and usefulness for safety. We, therefore, have developed a bioinspired robotic arm combining elements of rigid and soft robotics such that it exhibits the positive qualities of both, namely compliance and accuracy, while maintaining a low weight. This article describes the design of a robotic arm-wrist-hand system with seven degrees of freedom (DOFs). The shoulder and elbow each has two DOFs for two perpendicular rotational motions on each joint, and the hand has two DOFs for wrist rotations and one DOF for a grasp motion. The arm is pneumatically powered using custom-built McKibben type pneumatic artificial muscles, which are inflated and deflated using binary and proportional valves. The wrist and hand motions are actuated through servomotors. In addition to the actuators, the arm is equipped with a potentiometer in each joint for detecting joint angle changes. Simulation and experimental results for closed-loop position control are also presented in the article.
\end{abstract}

Keywords: inflatable robot, human-safe robot, assistive robot, bioinspired robot, pneumatic artificial muscle

\section{Introduction}

$\mathbf{R}$ OBOTS ARE BECOMING increasingly relevant and common in day-to-day life, ranging from simple wandering vacuum cleaners ${ }^{1}$ to tour guides ${ }^{2,3}$ and autonomous vehicles. ${ }^{4,5}$ As people continue to find uses for robots, we can only expect to see a continued increase in human-robot interaction. Consequently, human and animal safety have become a major concern around large and/or powerful robotic systems due to the generally damaging and unforgiving nature of colliding or interfering with one. ${ }^{6}$ Although collision prevention can help reduce the likelihood of an impact, such a method requires a detailed knowledge of the environment, ${ }^{7,8}$ which cannot be guaranteed in a human-populated area. Other strategies include introducing mechanical compliance in the $\operatorname{arm}^{9,10}$ and impedance control. Although the safety level can be increased with the compliance in this case, there always exists rigidity in the structure that may be still dangerous in certain applications. As a result, there has been a recent surge in research directed toward developing and implementing "soft robots," wherein systems are designed to be gentle and physically compliant so as to be harmless in the presence of people or delicate objects. ${ }^{11-15}$ This often involves using inflatable structures, ${ }^{16-18}$ actuator alternatives to the motor, ${ }^{19,20}$ or force feedback control systems. ${ }^{21,22}$

With current approaches, however, a common downside to being "soft" is also having a very limited load capacity. For example, Festo's Bionic Tripods and Bionic Handling Assistants, although very lightweight and agile, are depicted moving objects no heavier than small fruit and threedimensional (3D)-printed shapes. ${ }^{23-25}$ Those that are stronger, such as Otherlab's inflatable robots, are often too large

\footnotetext{
${ }^{1}$ Robotics Institute, Carnegie Mellon University, Pittsburgh, Pennsylvania.

${ }^{2}$ Department of Mechanical Engineering, Carnegie Mellon University, Pittsburgh, Pennsylvania.

${ }^{3}$ Department of Mechanical and Aerospace Engineering, Seoul National University, Seoul, Korea.

${ }^{4}$ Soft Robotics Research Center, Seoul National University, Seoul, Korea.
} 
for an in-home domestic robot. ${ }^{26}$ Another common drawback is the loss of accuracy in position control due to unaccounted deformations in the system and/or the inherently underdamped nature of a compliant actuation system. ${ }^{27}$ This can generally be corrected by slowing the system to near-quasistatic loading scenarios, but at the expense of operating time.

To address this apparent dichotomy between safety and effectiveness, we developed a bioinspired robotic arm (Fig. 1) that combines soft and rigid systems in a way that resembles the human arm in terms of structure, actuation, and degrees of freedom (DOFs). It consists of a lightweight skeleton made of carbon fiber composite tubes, 3D-printed rigid plastic joints, on-arm pneumatic artificial muscles (PAMs), and a servocontrolled underactuated hand. An external inflatable sleeve adds to the compliant nature of the air muscles by providing a layer of soft cushioning to dampen impacts.

The internal structure allows for a higher degree of kinematic accuracy than purely soft systems, and the use of lightweight materials and arm-mounted air muscles renders the arm lightweight as a whole and more physically compliant than purely rigid systems. Although maximizing force output was not a primary focus of this prototype, the internal structure also has the benefit of simply being able to add muscles for increased load capacity at the small sacrifice of a few grams per muscle and a bit of extra tubing. In other arms, increasing joint torque capabilities requires replacing motors or gear boxes, the approach of simply adding muscles allows the user to tune the arm to the required task at will. As a result,

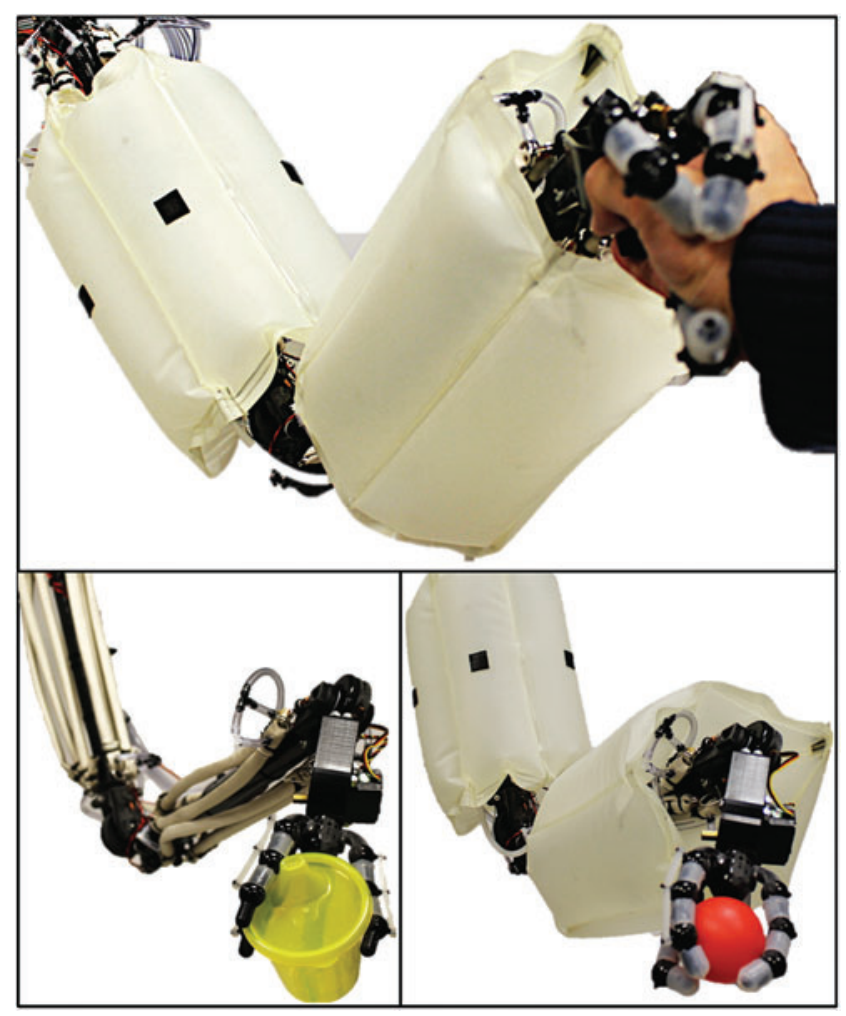

FIG. 1. Robotic arm composed of a lightweight rigid carbon fiber composite skeleton, soft inflatable sleeves, and PAMs, showing handling of various objects. PAMs, pneumatic artificial muscles. Color images available online at www.liebertpub.com/soro this "hybridized" arm exhibits a combination of properties often found in soft or rigid systems, exclusively.

Similar systems in this vein of research include the distributed macro-mini actuation system, ${ }^{28}$ which combines a rigid external structure with series elastic actuators for coarse movement, and an integrated motor in each joint for fine movement. ${ }^{29}$ As a result, it maintained a fair degree of mechanical compliance while still being accurate. Our system, although similar, diverges from this design so as to confine rigid elements to a simple, compact, and lightweight internal structure. We also seek to rely entirely on elastic actuators for both fine and coarse positioning to minimize mass and maximize safety, and extend our design to a seven DOFs system.

Taking a bioinspired approach has several other advantages. Considering the intended application for such a robot is in a domestic environment, where most tools and utilities are already optimized for human interaction, a human-like design will allow it to better interface with its surroundings. In addition, a robot might be frequently physically interacting with its human users, so giving it a more organic, relatable appearance and behavior would result in less emotional resistance to it, as compared with traditional designs (such as those of industrial robots). ${ }^{30-32}$

\section{Design}

\section{Arm}

One of the main design goals is to develop a robotic arm that can operate safely in the presence of people, while still being relatively strong and precise. In this context, "safe" is defined as not causing physical harm to a nearby entity, even in the event of a collision. We approached this goal for the robot arm and hand in two ways: using physically compliant mechanisms for actuation and designing the system with lightweight materials. This reduces the total inertia in the system and enables mechanical compliance, both of which are ideal for a safe robot. As such, in the event of a collision, the arm will deflect and impart a minimal amount of force to a person or object in its path.

Therefore, we wanted to use an actuator that was both lightweight and mechanically compliant. Consequently, series elastic actuators were a viable option, as they can exhibit both of these properties. ${ }^{27}$ Looking at pneumatic systems, since the compressible nature of air allows for inherently compliant systems, we decided to use McKibben-type ${ }^{33-35}$ air muscles because they are deformable, elastic, and physically lightweight. ${ }^{14,36,37}$

To minimize mass, we chose materials that had a high strength-to-weight ratio and that were easy to work with. For links, we used thin-walled carbon fiber rods due to their very high strength-to-weight ratio and suitable precut lengths. The joints were made of a 3D-printed lightweight photopolymer whose mechanical properties are comparable with acrylonitrile butadiene styrene (ABS) plastic. The 3D printing manufacturing process also had several key benefits, as is described in detail later. Heavy materials such as metal were avoided except wherein structurally necessary due to size and loading requirements, such as hollow joint pins. Figures 2 and 3 show the actual prototype of the robotic arm and its simplified diagram with Denavit-Hartenberg (DH) parameters, respectively. 


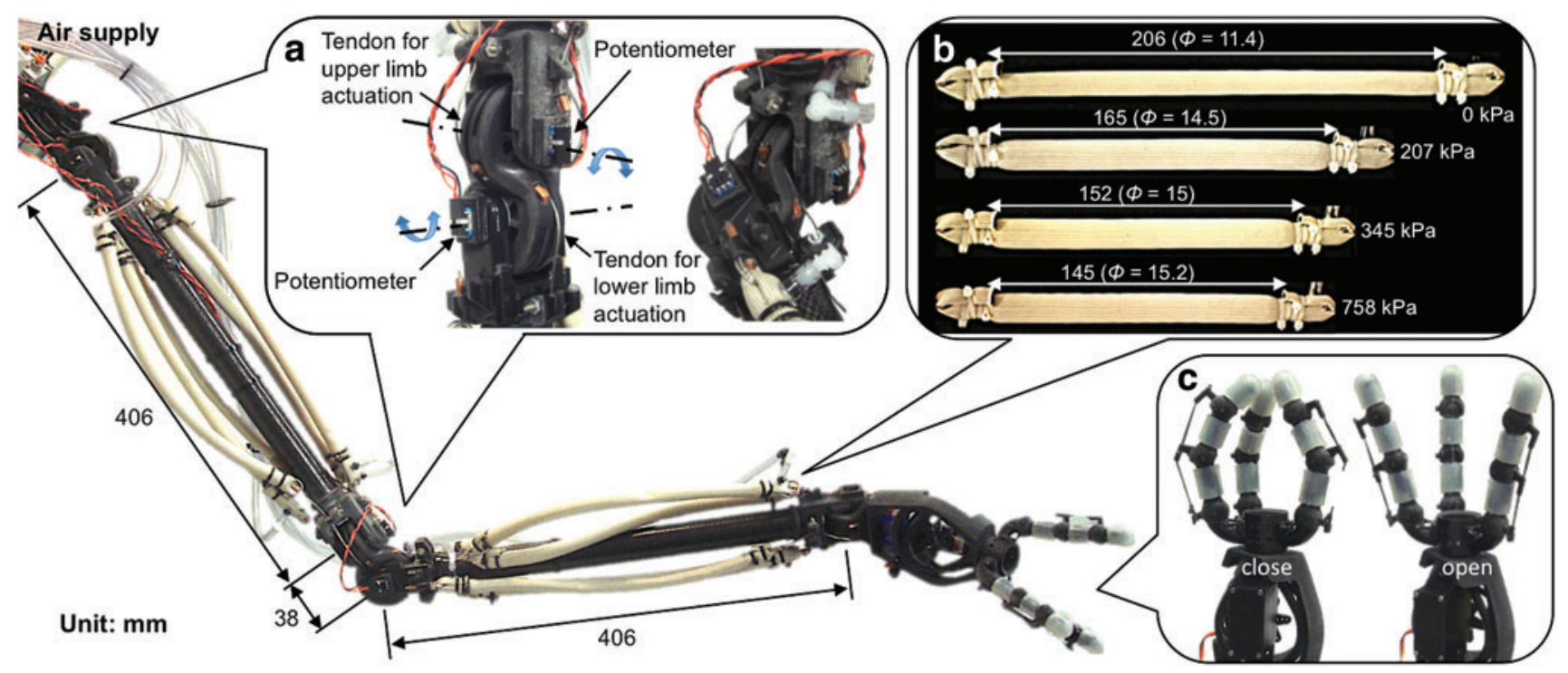

FIG. 2. Image of the robot arm prototype with details of major components. (a) 2 DOFs shoulder and elbow joints. (b) Custom-built PAM and its geometry changes with different actuation pressures. (c) Three-fingered underactuated robotic gripper (1 DOF) with its wrist joint (2 DOFs). DOF, degree of freedom. Color images available online at www .liebertpub.com/soro

\section{Joints}

To design the joints, we needed to first establish physical requirements, such as range of motion. We wanted the arm joints to ideally have a high range of motion, and given the nature of linear actuators, we were limited to the maximum stroke length of the air muscles. We also wanted to keep the distance from the rotational axis of the joint, which serves as moment arms for the muscles, constant to maximize torque output from the muscles, and avoids potential mechanical singularities. Thus, the arm length, moment length, and range of motion are mathematically linked:

$$
\begin{gathered}
C \times L_{c}=R \theta, \\
L_{A}=L_{C}+2 D .
\end{gathered}
$$

From these equations and the nature of the muscles we manufactured, we aimed for a $180^{\circ}$ range, resulting in air muscles with a $25.4 \mathrm{~mm}$ contraction region, a link length of $305 \mathrm{~mm}$, and a cable curvature of $19 \mathrm{~mm}$. We decided this was a reasonably large range that also kept the arm itself from becoming too large. It also allowed us to make the arm links coplanar since we did not need to worry about the links interfering with each other.
We wanted to use very few parts per joint to minimize assembly complexity and weight due to connectors. 3D printing was thus ideal because we could print highly complex parts as single units, rather than needing to assemble multiple simpler parts to create an equivalent whole. As such, each joint consists of only two major components: a disk and a fork. During actuation, a disk, attached to the end of a link by an interface extending from its cylindrical surface, rotates about its axis between the two fingers of a supporting fork, which is fixed to a second link. The disk is actuated by a pair of antagonistic air muscles, positioned along the same link as the fork, through steel cables wound around the disk.

To achieve a $180^{\circ}$ range, the cables needed to wrap at least $180^{\circ}$ around the disk, otherwise the cable would approach a singularity, causing the moment length to approach zero and the disk to stop rotating. One possible solution was to wrap the cables on either side of the extension connecting the disk to its link, but separating the cables by a large amount from the joint's plane of rotation would produce an undesirably large off-axis moment. Instead, we routed the cables side-by-side through the disk itself, as this minimized the off-axis moment and could still be manufactured by our $3 \mathrm{D}$ printer. The first four DOFs in the arm were achieved by printing two double-linked versions of this joint, wherein the disk component now consisted of a pair of intersecting disks offset from each other by $90^{\circ}$, as shown in Figure 2 a.
FIG. 3. Free-body diagram of robot arm design with Denavit-Hartenberg conventions, showing two rotational joints on each of three joints.

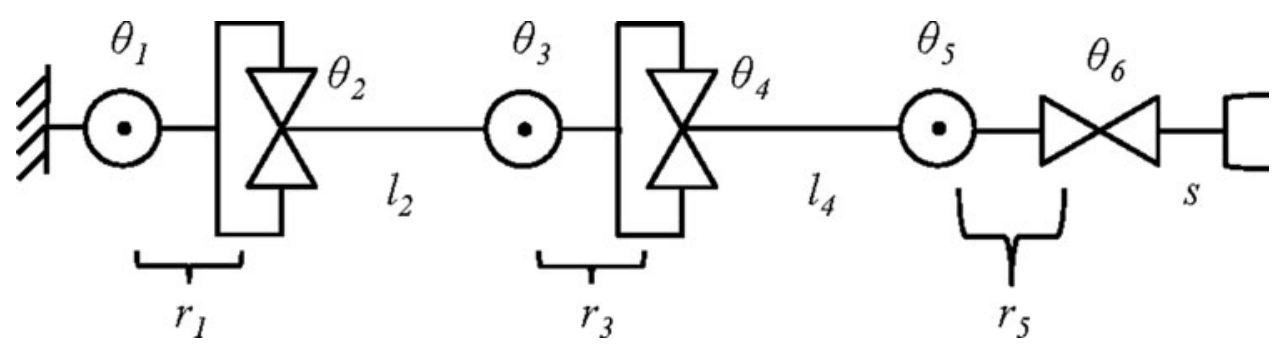


Consequently, two DOFs could be achieved with only three major parts.

The joint angles are sensed through a potentiometer mounted to each joint. Each disk features a hexagonal hole about its axis of rotation into which a hexagonal aluminum pin with rounded ends (for smooth rotation about the fork) is inserted, serving as a hinge that rotates relative to the fork but remains fixed relative to the disk. By press-fitting the rotor of a potentiometer into a hole through the axis of the pin and fastening the potentiometer base to a square housing in the fork, we could determine the angle of each joint by bijectively mapping the output voltage from the potentiometer to the angular position of the disk joint relative to the fork.

To further minimize the overall part count, the muscles actuating a disk joint on one end of an arm link are supported by mounts on the fork on the opposite end. This is possible because forks mounted on the same arm link are offset by $90^{\circ}$, allowing for two pairs of air muscles to be positioned along each arm link.

The fifth DOF, near the hand, is done by a single-disk joint and actuated by muscles mounted along the forearm. Position can again be detected using a potentiometer. The sixth and final DOF in the arm, closest to the hand, is a wrist-like twisting joint actuated by a servo.

\section{Actuators}

The arm is actuated by custom-built PAMs, which follow a McKibben-type design wherein an expanding bladder causes a braided mesh sleeve to contract along its axis. Instead of using discrete bladder and mesh components, however, we used a fiberglass-reinforced mesh tube with a layer of stretchable silicone bonded to the inner surface (Silicone Flex Glass; Techflex, Inc.). The muscles were fabricated by inserting a pneumatic plug into one end of the tube and an elbow connector inserted through the tube wall on the other end. These pieces were held in place by metal-reinforced ties. Excess tubing on each end was folded over and tied down to form loops, both of which house a metal split ring onto which a swaged cable was fastened. Figure $2 b$ shows the actuation behavior of our pneumatic muscle with different air pressures. Muscles were used either singularly or in bundles, depending on the loads experienced at each joint.

\section{Hand}

A three-fingered robotic hand was developed and attached to the lower arm, as shown in Figure 2c. Each finger was made of a 3D-printed rigid plastic material covered by soft silicone skin. The actuation of the hand was achieved by a tendon-driven underactuated system. An active tendon, located on the volar side of each finger and connected to a servo motor, induces finger-flexion motion, and an elastic passive tendon on the dorsal side induces finger-extension motion. The fingers also include fiber optic strain sensors for force and tactile sensing. Details on the hand can be found in our previous work. ${ }^{38}$

\section{Inflatable sleeves}

Although the air muscles allow for mechanically compliant actuation, an exposed skeletal structure could still result in high-impact forces if collided with directly, due to its ri- gidity. Soft sleeves comprised polyurethane sheets fused through an impulse heat sealer (MP-40; Midwest Pacific) into inflatable segments (i.e., air chambers), thus enveloped the structure, as shown in Figure 4. Each sleeve was divided into five segments (Fig. 5) to easily form a segmented toroidal structure around the arm, as shown in Figure 6. The segments were pressurized to $\sim 20 \mathrm{kPa}$ through a precision pressure regulator (R-800-30; Airtrol Components, Inc.), providing $3-5 \mathrm{~cm}$ of compressible padding.

\section{Control hardware}

For simple actuation, a bank of three-way ON-OFF air valves (MME-31PES; Clippard) were used to direct airflow into and out of the muscles. Each valve, when in the ON state, allows pressurized air to flow into a single air muscle, causing it to inflate. In the OFF state, a spring-return mechanism closes the connection to the inlet and opens the connection from the muscle port to the exhaust, allowing the muscle to deflate. When coupled with air flow reducer valves to lower the flow rate and, hence, the rate of muscle inflation and deflation, this setup allows for position control through a sliding mode (SM) controller.

For faster, more stable, and more precise control, a set of pneumatic proportional valves (MD Pro; Parker) was added to the system in series with the binary valves. Unlike the binary valves, wherein the valve aperture is only either completely open or completely closed, proportional valves can achieve intermediary states as a function of the input voltage, causing the flow rate of air passing through the valve to change accordingly. This allows for the use of a proportional-integral-derivative (PID) controller to control muscle inflation and deflation. Implementing this type of control on a pair of antagonistic muscle pairs permits accurate positioning of an arm joint. A diagram of the configuration of the valves is shown in Figure 7.

The bank of binary valves were controlled through a set of mechanical relays (16 Relay Board), with one relay for each valve. A diode was inserted across the terminals of the binary valves to handle the counter-electromotive force produced by the actuating valve solenoids, which would

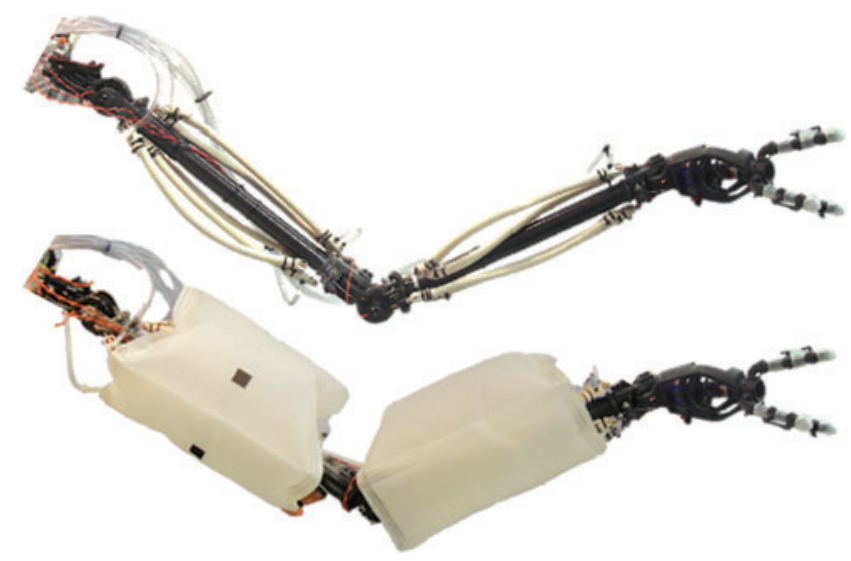

FIG. 4. Arm prototype made of rigid skeleton and pneumatic muscles before (top) and after (bottom) installing inflatable sleeves. Color images available online at www .liebertpub.com/soro 

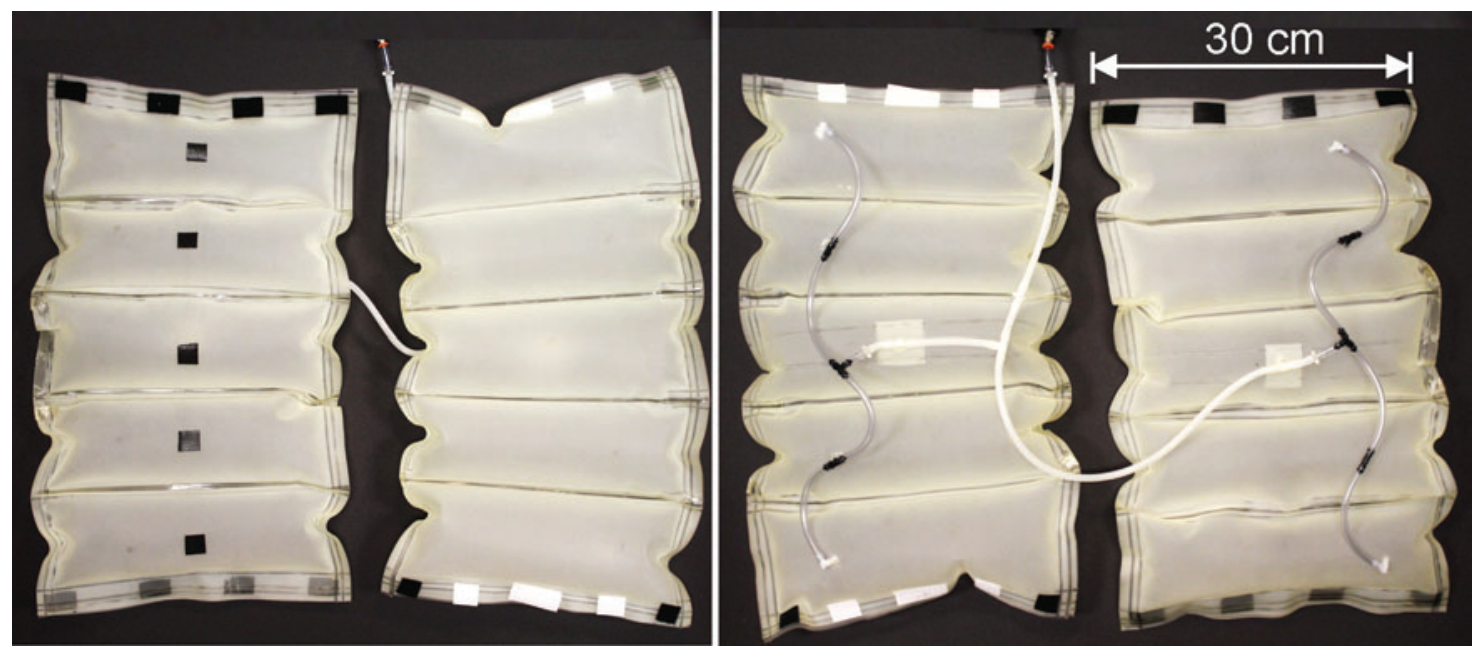

FIG. 5. Inflatable sleeves segmented with multiple air chambers to be easily wrapped around arms: Outside (left) and inside with pneumatic tubing (right) of sleeves. Color images available online at www.liebertpub.com/soro

otherwise shut down the relay board. The proportional valves were controlled by dual motor drivers (ROB-09457; Sparkfun), wherein each driver controlled the inflating and deflating valves on one side of a joint. All valves were controlled by a microcontroller (Leonardo, Arduino), which accepted position commands and power from a connected computer through a universal serial bus (USB) connection. The microcontroller implemented the feedback controller by sending commands to the relay board through an interintegrated circuit communication bus, and to the motor drivers through pulse-width-modulation (PWM) signals. Feedback was achieved using the potentiometers (COM-09288; Sparkfun) embedded in the joints, where the variable electrical re-

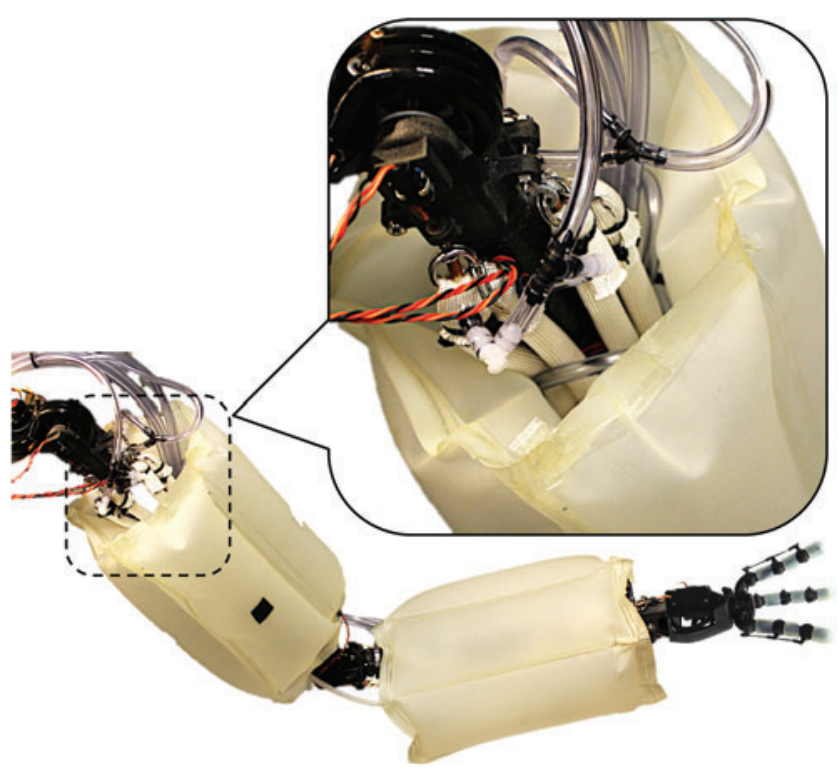

FIG. 6. Inflatable sleeves surrounding skeleton and pneumatic muscles for impact absorption, which help protecting humans interacting with the robot as well as the mechanical and electrical components of the robot. Color images available online at www.liebertpub.com/soro sistance was read by the microcontroller and converted into a joint angle. The valves and relay board were powered by an $11.5-\mathrm{V}$ power supply, and the microcontroller, motor driver logic circuits, and potentiometers were powered by a $5-\mathrm{V}$ power USB supply from a connected computer.

The wrist and hand servos were powered by the $11.5-\mathrm{V}$ power supply and controlled through PWM signals from the microcontroller.

\section{Characterization}

\section{Actuator}

The custom-built PAMs were characterized using a motorized materials test stand (ESM301, Mark-10) and a single-axis load cell (STL-50; AmCells). Figure 8 shows the characterization result of a single muscle for its contraction force and length. During the test, the air pressure was varied from 0 to $758 \mathrm{kPa}$. At the maximum air pressure, the muscle was able to contract up to $\sim 23 \%(47 \mathrm{~mm})$ of its original length with no load and to generate a pulling force up to $190 \mathrm{~N}$ with negligible displacement.

\section{Sensor}

The joint angle sensors (potentiometers) were validated using a digital goniometer ( $i$ GAGING), as shown in Figure 9. The plot shows the calibration result of a single sensor by measuring voltage outputs when $5-\mathrm{V}$ supply voltage was applied. Four sets of output voltages were recorded every $5^{\circ}$ between $-80^{\circ}$ and $80^{\circ}$. The result showed a linear relationship between voltage output and joint angle. The sensitivity was $\sim 23 \mathrm{mV} /{ }^{\circ}$.

\section{Workspace Analysis}

Using pneumatic muscles as a form of actuation yields many benefits over traditional actuators, such as electromagnetic motors, mainly a far superior power-to-weight ratio ${ }^{33-35}$ as well as reduced financial cost. However, the capabilities and control strategies of this class of actuators are poorly understood compared with traditional motors. Even so, the desire for 

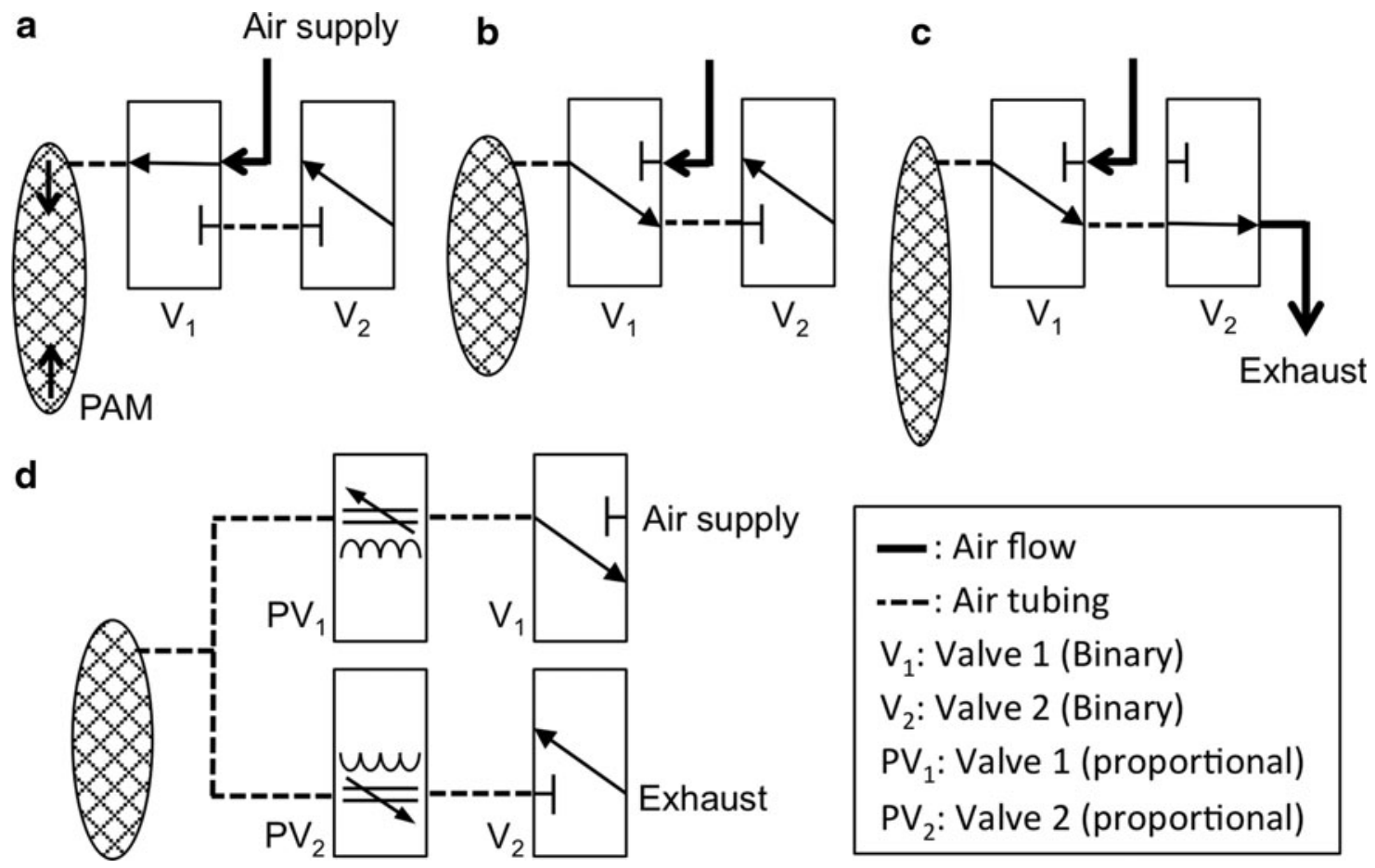

FIG. 7. Muscle behaviors with different valve configurations. (a) Contraction (air injection): V1-ON, V2-OFF. (b) Hold (air is neither injected nor released.): V1-OFF, V2-OFF. (c) Relax (air release): V1-OFF, V2-ON. (d) Proportional valves were inserted between the muscle and the binary valves for proportional control.

low-cost, low-mass, high-precision manipulators is desired, serving as motivation to investigate their use on traditional robotic arms. The objective of this portion of the article is to formulate the kinematics and kinetostatics of the proposed arm design to positively demonstrate the feasibility of using this class of actuators on a traditional robotic arm. As a result, the design, fabrication, analysis of dynamic behaviors, and implementation of control strategies are further investigated and discussed in the subsequent portions of the article.

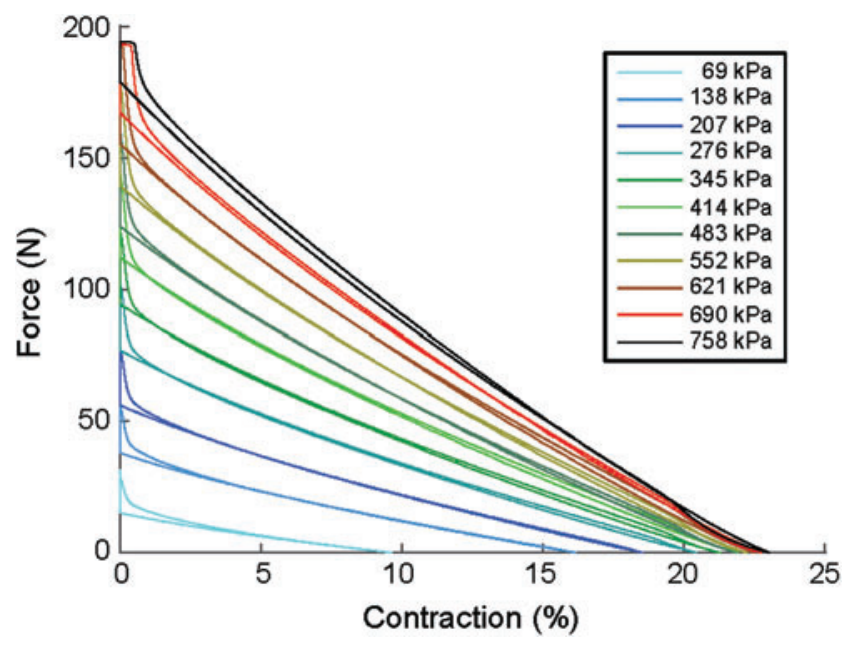

FIG. 8. Characterization result of custom-built PAM showing the maximum contraction force of $190 \mathrm{~N}$ and the maximum contraction rate of $23 \%$ at $758 \mathrm{kPa}$. (Although the muscle was able to tolerate higher pressure for higher force, the experiment was stopped at $758 \mathrm{kPa}$ for safety.) Color images available online at www.liebertpub.com/soro

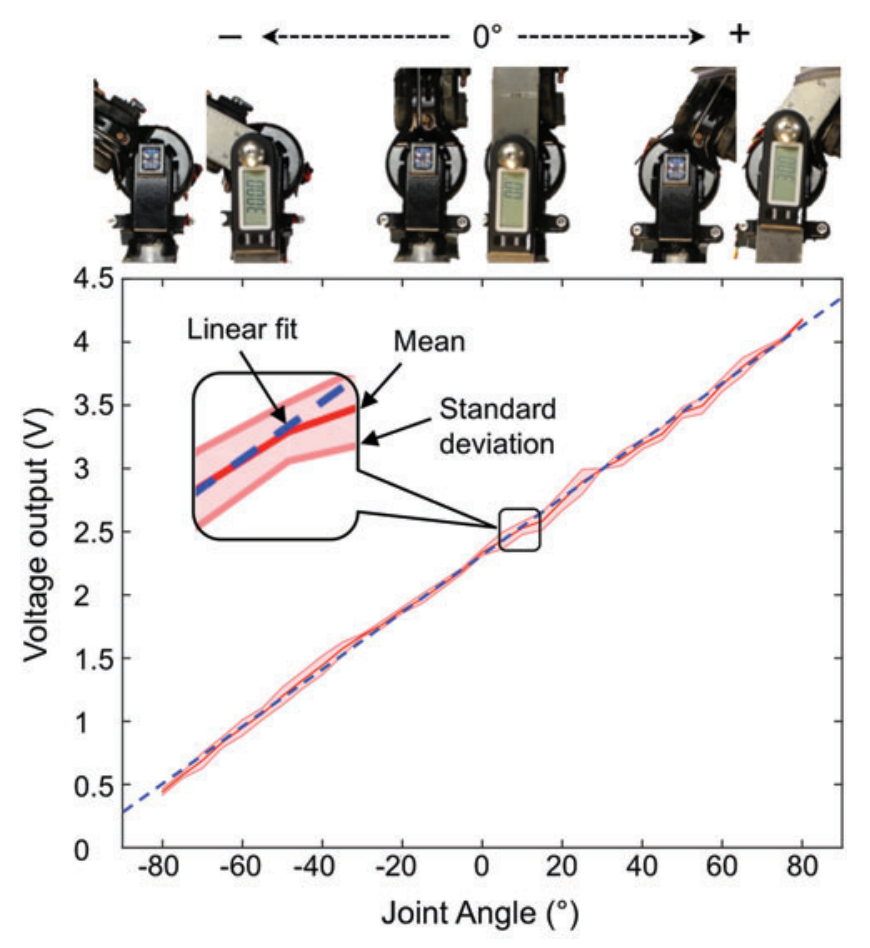

FIG. 9. Joint angle sensor (potentiometer) calibration setup using a digital goniometer (top) and results showing a linear relationship between joint angle and voltage output (bottom). Color images available online at www.liebertpub.com/soro 


\section{Kinematic representation}

The robotic arm (manipulator), represented in Figure 10a, can be kinematically defined by its DH parameters, given in Table 1; the number of muscles actuating each joint, $n$, is also included in the table for convenience.

From the DH parameters we can explicitly express the kinematic mapping, $T_{i}^{i+1}$, between joints as transformations on homogeneous six vectors, $\xi_{i}$ (i.e., screw displacements or twists):

$$
\begin{gathered}
\xi_{i}\left(\theta_{i}\right)=\left[\begin{array}{llllll}
0 & 0 & \theta_{i} & 0 & 0 & 0
\end{array}\right]^{T} \quad \in \operatorname{se}(3), \\
T_{i}^{i+1}\left(\xi_{i}\right)=\left[\begin{array}{cc}
\mathbf{R}_{i}^{i+1} & \mathbf{t}_{i}^{i+1} \\
0_{1 \times 3} & 1
\end{array}\right] \quad \in \operatorname{SE}(3), \\
\mathbf{R}_{i}^{i+1}=\left[\begin{array}{ccc}
c_{\theta_{i}} & -s_{\theta_{i}} c_{\alpha_{i}} & s_{\theta_{i}} s_{\alpha_{i}} \\
s_{\theta_{i}} & c_{\theta_{i}} c_{\alpha_{i}} & -c_{\theta_{i}} s_{\alpha_{i}} \\
0 & s_{\alpha_{i}} & c_{\alpha_{i}}
\end{array}\right] \in S O(3), \\
\mathbf{t}_{i}^{i+1}=\left[\begin{array}{lll}
a_{n} c_{\theta_{i}} & a_{n} s_{\theta_{i}} & d_{i}
\end{array}\right]^{T} \in \mathbb{R}^{3} .
\end{gathered}
$$

It is worth to note that the rotation matrix, $\mathbf{R}_{i}^{i+1}$, relating adjacent joints simply defines the principle coordinate axes for the new coordinate frame relative to the subsequent coordinate frame. Similarly, the translation vector, $\mathbf{t}_{i}{ }^{i+1}$, locates the new coordinate frame relative to the subsequent coordinate frame:

$$
\begin{gathered}
\mathbf{R}_{i}^{i+1}=\left[\begin{array}{ll}
\hat{X}_{i}^{i+1} \hat{Y}_{i}^{i+1} \hat{Z}_{i}^{i+1}
\end{array}\right], \\
\mathbf{t}_{i}^{i+1}=\left[\begin{array}{lll}
x_{i}^{i+1} & y_{i}^{i+1} & z_{i}^{i+1}
\end{array}\right]^{T} .
\end{gathered}
$$

We can compute the kinematic mapping between any two joints relative to one another using the following relationships:

$$
\begin{gathered}
T_{i}^{j}=T_{i}^{i+1} T_{i+1}^{i+2} \cdots T_{j-1}^{j}, \\
T_{i}^{j}=\left\{\begin{array}{lll}
\boldsymbol{I}_{4} & \text { if } i=j . \\
\left(T_{j}^{i}\right)^{-1} & \text { if } j>i
\end{array}\right.
\end{gathered}
$$

We can apply domain constraints to the joint angles in correspondence with their physical joint limits:

$$
\boldsymbol{\theta}:=\left\{\theta_{i} \mid \theta_{i} \in\left[-\frac{\pi}{2}, \frac{\pi}{2}\right] \forall i \in[1,2, \cdots, 5]\right\} .
$$

We can then construct the maximum reachable workspace, $\mathcal{W S}_{m}$, of the manipulator with constrained joint limits. This is achieved by densely sampling manipulator poses from the joint angle domain space, and then computing the alphashape boundary of the resulting set of end-effector positions. The result is shown in Figure $10 \mathrm{~b}$ for a sample size of 10,000 poses:

$$
\partial \mathcal{W S}_{m}=\alpha-\operatorname{Shape}\left(\left\{t_{1}^{5} \mid \forall \theta_{i} \in\left[-\frac{\pi}{2}, \frac{\pi}{2}\right]\right\}\right) .
$$
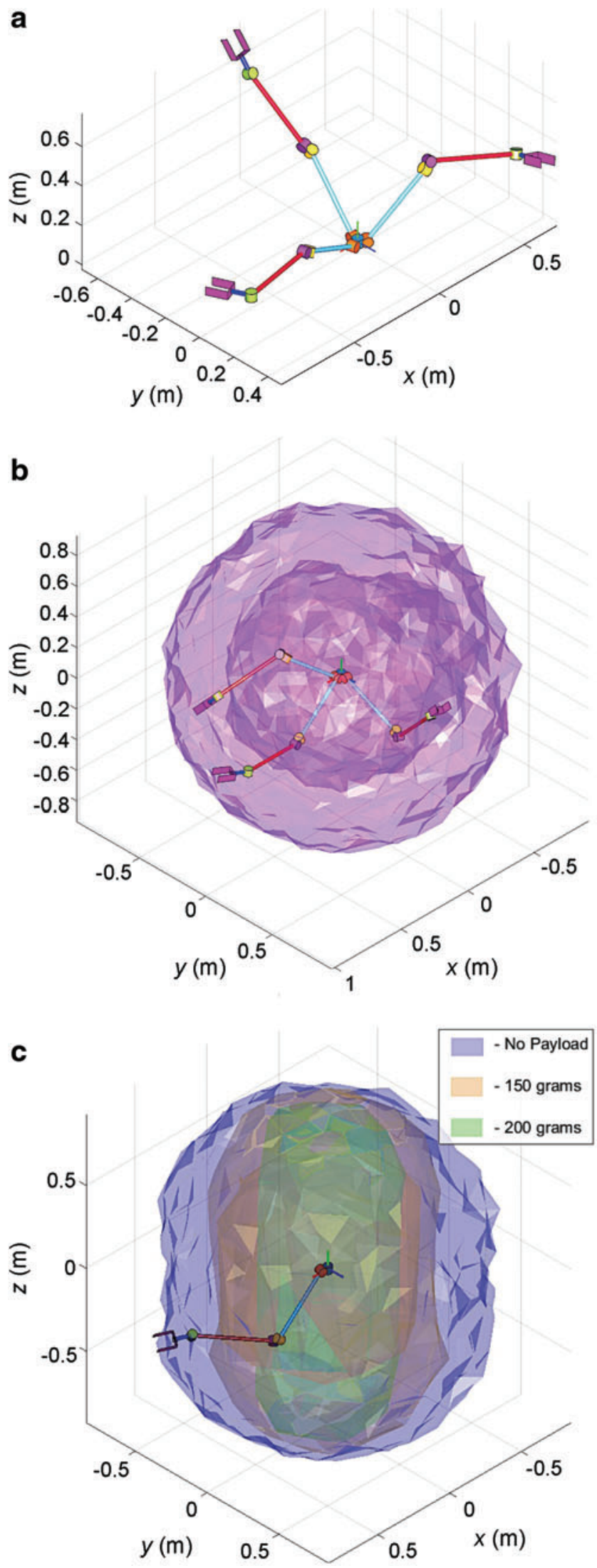

FIG. 10. Workspace analysis results. (a) Sample possible poses of the robotic arm. (b) Reachable workspace boundary with joint limits based on kinematic representation. (c) Reachable workspaces for various payloads based on kinetostatic analysis. Color images available online at www.liebertpub.com/ soro 
Table 1. Denavit-Hartenberg Parameters

\begin{tabular}{lccccc}
\hline Link & $a(\mathrm{~cm})$ & $\alpha(\mathrm{rad})$ & $d(\mathrm{~cm})$ & $\theta$ & $\mathrm{n}$ \\
\hline 1 & 3.8 & $\pi / 2$ & 0 & $\theta_{1}$ & 1 \\
2 & 40.6 & $-\pi / 2$ & 0 & $\theta_{2}$ & 3 \\
3 & 3.8 & $\pi / 2$ & 0 & $\theta_{3}$ & 3 \\
4 & 40.6 & $-\pi / 2$ & 0 & $\theta_{4}$ & 2 \\
5 & 8.9 & 0 & 0 & $\theta_{5}$ & 1 \\
\hline
\end{tabular}

\section{Kinetostatics}

These data in Figure 8 were used to bijectively map the muscle contraction to the maximum muscle force (this analysis uses the results for a working pressure of $760 \mathrm{kPa}$ ):

$$
f_{\text {max }}(C): \mathbb{R} \rightarrow \mathbb{R} .
$$

Each joint has an identical radius, $r$, that the muscle tendons act on. For any manipulator pose defined by $\boldsymbol{\theta}$ we can compute the change in muscle length as:

$$
\Delta l:=\left\{\Delta l_{1}, \Delta l_{2}, \cdots, \Delta l_{5}\right\}=\left\{r \theta_{i} \mid \forall \theta_{i} \in \boldsymbol{\theta}\right\}
$$

Given a constant starting muscle length, $l_{0}$, the relative change in muscle length is given as:

$$
\begin{gathered}
C_{i}=\frac{\Delta l_{i}}{l_{0}} \\
C:=\left\{C_{1}, C_{2}, \cdots, C_{5}\right\}
\end{gathered} .
$$

Using the relationship between muscle contraction and maximum applied force given in Equation (13), and the number of muscles actuating each joint, $n=\left\{n_{1}, n_{2}, \ldots, n_{5}\right\}$, given in Table 1, we can reformulate the mapping to be a function of the joint angle and as a result construct the set of maximum forces that can be applied at each joint for a given pose:

$$
\begin{gathered}
f_{\max }(\theta): \quad \mathbb{R} \rightarrow \mathbb{R} \\
f_{\text {max }}(\boldsymbol{\theta}): \quad \mathbb{R}^{5} \rightarrow \mathbb{R}^{5} \\
f_{\text {max }}(\boldsymbol{\theta})=\frac{l_{0}}{r} \times\left\{f_{\text {max }}(\boldsymbol{C}) \circ \boldsymbol{n}\right\}
\end{gathered},
$$

where $x$ and $\circ$ are the Cartesian product and Hadamard product operators, respectively.

Finally, using the relationship between torque about an axis and a force applied to that axis through an orthogonal lever, $\tau=r \times f=r f$, we can express the ordered set of maximum allowable joint torques for a given pose defined by $\boldsymbol{\theta}$ :

$$
\begin{gathered}
\tau_{\max }(\boldsymbol{\theta}): \mathbb{R}^{5} \rightarrow \mathbb{R}^{5} \\
\tau_{\max }(\boldsymbol{\theta})=r \times f_{\max }(\boldsymbol{\theta})=l_{0} \times f_{\max }(\boldsymbol{C}) \circ \boldsymbol{n} .
\end{gathered}
$$

Static joint loads. For any given kinematic mapping, the corresponding Jacobian is computed through outer product partial differentiation with respect to the joint angles:

$$
\begin{gathered}
{ }_{i}^{j} J=\left[\begin{array}{c}
\frac{\partial \mathbf{t}_{i}^{j}}{\partial \theta_{i}^{j}} \\
\mathbf{Z}_{i}^{j}
\end{array}\right] \in \mathbb{R}^{6 \times(j-i+1),} \\
\mathbf{Z}_{i}^{j}:=\left[\begin{array}{lll}
\hat{Z}_{i}^{i} \hat{Z}_{i}^{i+1} & \cdots & \hat{Z}_{i}^{j}
\end{array}\right] \in \mathbb{R}^{3 \times(j-i+1)} .
\end{gathered}
$$

By the principal of virtual work, the joint torques on a serial manipulator are given by:

$$
\tau_{i}^{j}={ }_{i}^{j} J^{T} w_{i}
$$

where $w_{i}$ is the wrench induced on joint $i$ due to the mass of link $i$ acting through its center-of-mass in the direction of the gravity vector, $\overrightarrow{f_{i}}=m_{i} \vec{g}$. The joint torques for a given pose are given by:

$$
\begin{gathered}
\tau_{b}(\theta)={ }_{1}^{5} J^{T} w_{\text {payload }}+\sum_{i=1}^{5}{ }_{i}^{j} J^{T} w_{i} \in \mathbb{R}^{5}, \\
\tau_{b}(\theta):=\left[\begin{array}{llll}
\tau_{b, 1} & \tau_{b, 2} & \cdots & \tau_{b, 5}
\end{array}\right]^{T},
\end{gathered}
$$

where $w_{\text {payload }}$ is the external wrench acting on the endeffector by some payload.

Static reachable workspace. We now know the joint torques for any pose (and any end-effector payload) given by Equation (21), as well as the maximum allowable torques for any pose given by Equation (17). So for the set of poses, we can find the subsets of poses that satisfy the maximum joint torque constraints, effectively producing static load reachable workspaces.

The set of feasible poses for a given payload is:

$$
\boldsymbol{\theta}^{*}\left(w_{\text {payload }}\right)=\left\{\boldsymbol{\theta} \mid \tau_{b, i} \leq \tau_{\text {max }, i} \forall i \in[1,5]\right\} .
$$

A static workspace, $\mathcal{W S}^{s}$, is the set of reachable positions described by the set of feasible poses. This is equivalent to the set of end-effector positions for each pose in the set of feasible poses:

$$
\mathcal{W S}_{\text {payload }}^{s}=\mathbf{t}_{1}^{5}\left(\theta_{\text {payload }}^{*}\right)
$$

Taking the alpha-shape of $\mathcal{W S}^{s}$ produces the boundary of the static workspace, which can be easily visualized. The results for several payloads are shown in Figure 10c.

The static reachable workspaces for small payloads, although certainly not equivalent to $\mathcal{W S}_{m}$, seem to be useable. We can conclude that this type of manipulator is worth investigating further for low payload tasks such as pick-andplace of small parts or personal assistance, such as cooking and administering medication.

\section{Control}

\section{Simulation}

Based on the result shown in Figure 8, the contraction force $F$ can be fitted to a function of input air pressure $(p)$ and contraction $(\Delta l)$ as

$$
F=1.14+1.87 p-1.90 \Delta l-0.08 p \Delta l+0.08 \Delta l^{2} .
$$

For control simulation, the following assumptions were used for modeling the behavior of the pneumatic muscle:

- Fibers in the muscle are inextensible.

- Coulomb friction in the muscle is negligible, resulting in no hysteresis.

- No explicit time dependence is considered. 
The following assumptions were also used, simulating the mechanical system of the arm:

- The arm structure is rigid.

- The mass and the inertia of the pneumatic muscles are negligible.

- The reflected inertia by the muscles to the joints is negligible.

- Viscous friction is the only source of friction and assumed to be linear with respect to joint velocity.

- The muscle length is strictly and antagonistically coupled to the angle of its corresponding joint.

Based on the mentioned assumptions, simulation of the dynamic system of the arm was conducted. Two approaches were used to test the dynamics: an Euler method approach through MATLAB and a Runge-Kutta integrator in the ODE-45 solver through Simulink.

In the first approach, the screw theory was used to derive the Euler-Lagrange equations of motion of the five DOFs arm shown in Equation (26). Both methods used the same equations of motion.

$$
\begin{gathered}
M(\theta) \ddot{\theta}+C(\theta, \dot{\theta}) \dot{\theta}+G(\theta)+F(\dot{\theta})=\tau, \\
F=\operatorname{sign}(P)\left(1.14+1.87|P|-1.90 L-0.81|P| L+0.08 L^{2}\right),
\end{gathered}
$$

where $\theta$ and $\dot{\theta}$ are the actual angle and the angular velocity of the system, respectively. $M$ is the mass tensor matrix, $C$ is the centripetal Coriolis matrix, $G$ is the gravity vector, $F$ is the viscous friction vector, and $\tau$ is the torque applied to the system.

We tested three different controllers in simulation: PID, SM, and robust integral of the sign of the error (RISE). We were interested in the stability and tracking properties of the system. Theoretically, using Lyapunov analysis in the dynamics of Equation (26) and without considering feedback linearization of the torque input, the PID controller is locally stable/tracking, SM is globally exponentially stable/tracking, and RISE is semiglobal asymptotically stable/tracking. ${ }^{39}$ The assumption for SM is that the actuator used provides infinite frequency, which in practice is impossible. Hence, SM will always yield a uniformly ultimately bounded (UUB) result instead of global exponential tracking. The RISE controller is basically a PID controller combined with some robustness properties of SM, without the disadvantage of the intrinsic discontinuity of SM. To solve this problem, the integral of the SM term is used.

For the simulation we used two metrics. The first one was a tracking metric for which step responses at different angles were tested. The second was the tracking of a sinusoid.

When comparing the step responses of the PID, RISE, and SM controllers in Figure 11a, c, and e, respectively, it can be observed that the response of the PID and the RISE controllers resembles each other. This is because both have a similar structure. Not a big difference can be observed in this metric, except for oscillations possibly due to the effects of the sign function at angles wherein the actuation bandwidth is limited. The SM step shows a higher rise time and virtually no overshoot. The cause of this is the infinite actuator frequency assumption of SM. Owing to the quasi-static nature of our muscle model, the simulation yields an UUB result as expected. The transient exponential convergence and the chatter at steady state are evidence of this.

When comparing the sinusoid tracking behavior of the PID, RISE, and SM controllers in Figure 11b, d, and $\mathrm{f}$, respectively, it can be observed that the PID response and the RISE response again resemble each other. However, this time, the PID controller does not reach the peaks of the sinusoid, whereas RISE does. We introduced a time delay to the SM simulator to compensate for the high frequency characteristic of our muscle model. This time, since the result remained so close to the region of convergence, the undesired effects of a UUB result were evident.

An underlying assumption of SM is infinite frequency of the actuators. An underlying assumption of the PID controller is infinite gain. RISE has both assumptions, but the integral of the sign of the error was given significantly less gain than the rest of the controller. Therefore, the infinite gain assumption dominates the application of the controller in this system. In simulation, it is shown that SM has better performance than PID and RISE. This is because the high frequency assumption was more idealized in simulation than the high-gain assumption. For PID, we initially select gains taking the physical actuation limitation into account. We use the Ziegler-Nichols methodology and then fine-tune it to our particular case. Hence, degraded performance due to gain is accounted for. However, for SM, although a delay was introduced in the controller, it did not account for certain lags in the physical system, which would include characterization of PWM, internal dynamics of the valves, and head loss. These losses are more coupled with the frequency of the system rather than the gain.

\section{Experiments}

To understand how the system behaves, we needed to implement controllers to observe its behavior and determine what level of accuracy is achievable with the system. As such, our initial tests involved implementing two different controllers: SM and PID. For both controllers, we commanded one of the arm joints to move to various positions, with the angular displacement being sent from the microcontroller to the connected computer over a USB-serial connection. The data were time stamped and recorded on the computer using RealTerm, a serial terminal program.

In our experiments, a controller that combines the SM and PID controllers was developed, resulting in a fast overall response time, relatively high damping, and low steady-state error. Over large distances (defined by states in which the combined results of the proportional and derivative gains result in a voltage more than the threshold proportional valve voltage), a PID controller is utilized until the distance to the target is small (when the combined effects of the proportional and derivative gains are less than the threshold voltage), where a SM controller with a low gain is used instead. The SM controller produces a voltage slightly more than the threshold voltage, resulting in minimal flow through the proportional valves. In addition, the SM controller does not operate continuously, but instead pulses the proportional valves at a $33 \%$ duty cycle as though they were binary valves, further reducing the effective flow rate.

The combined effects of these elements allow the controller to converge to its target significantly faster than either 

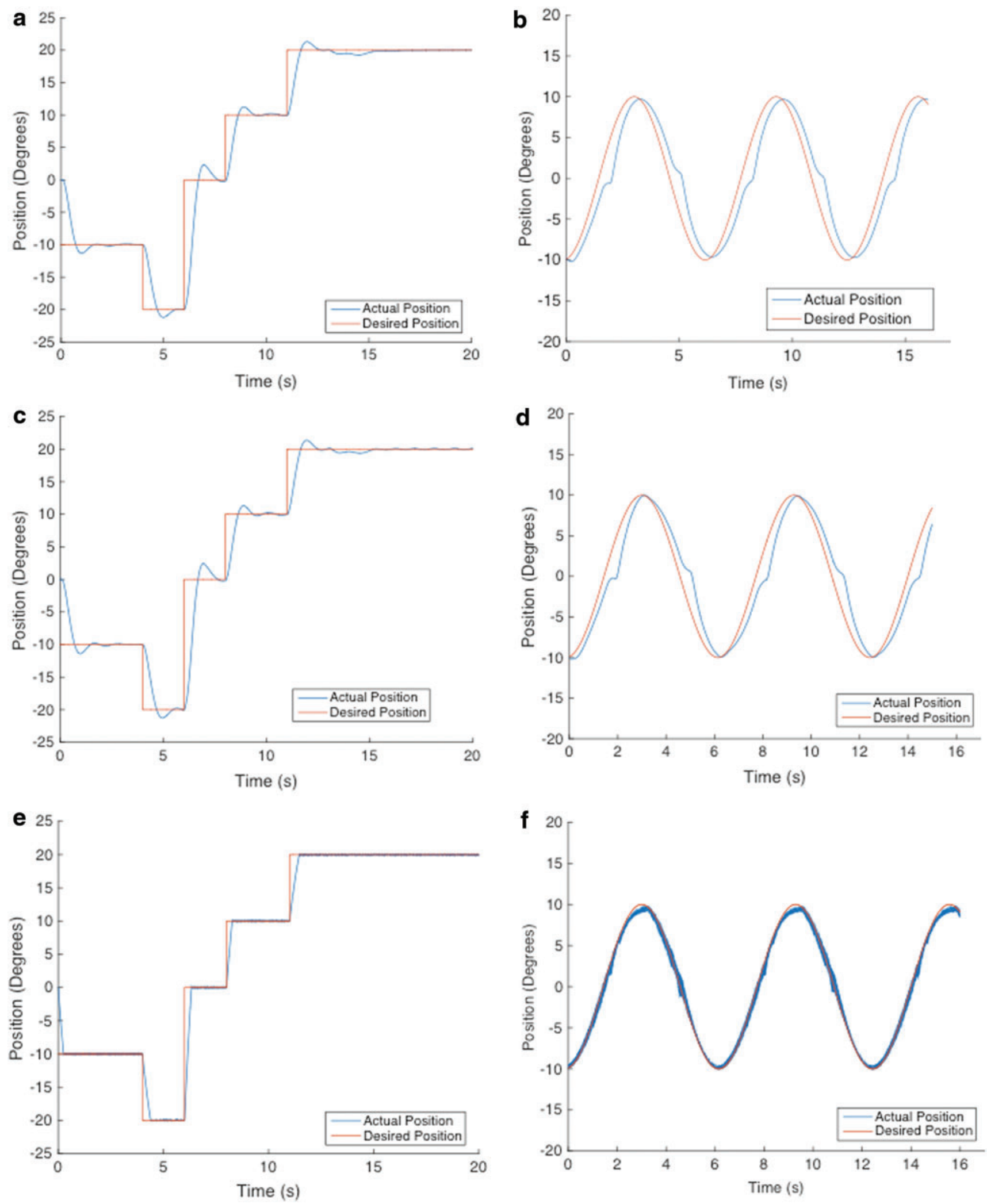

FIG. 11. Simulation results of feedback control. (a) PID multistep response. (b) PID sinusoidal response. (c) RISE multistep response. (d) RISE sinusoidal response. (e) SM multistep response. (f) SM sinusoidal response. PID, proportionalintegral-derivative; RISE, robust integral of the sign of the error; SM, sliding mode. Color images available online at www .liebertpub.com/soro 

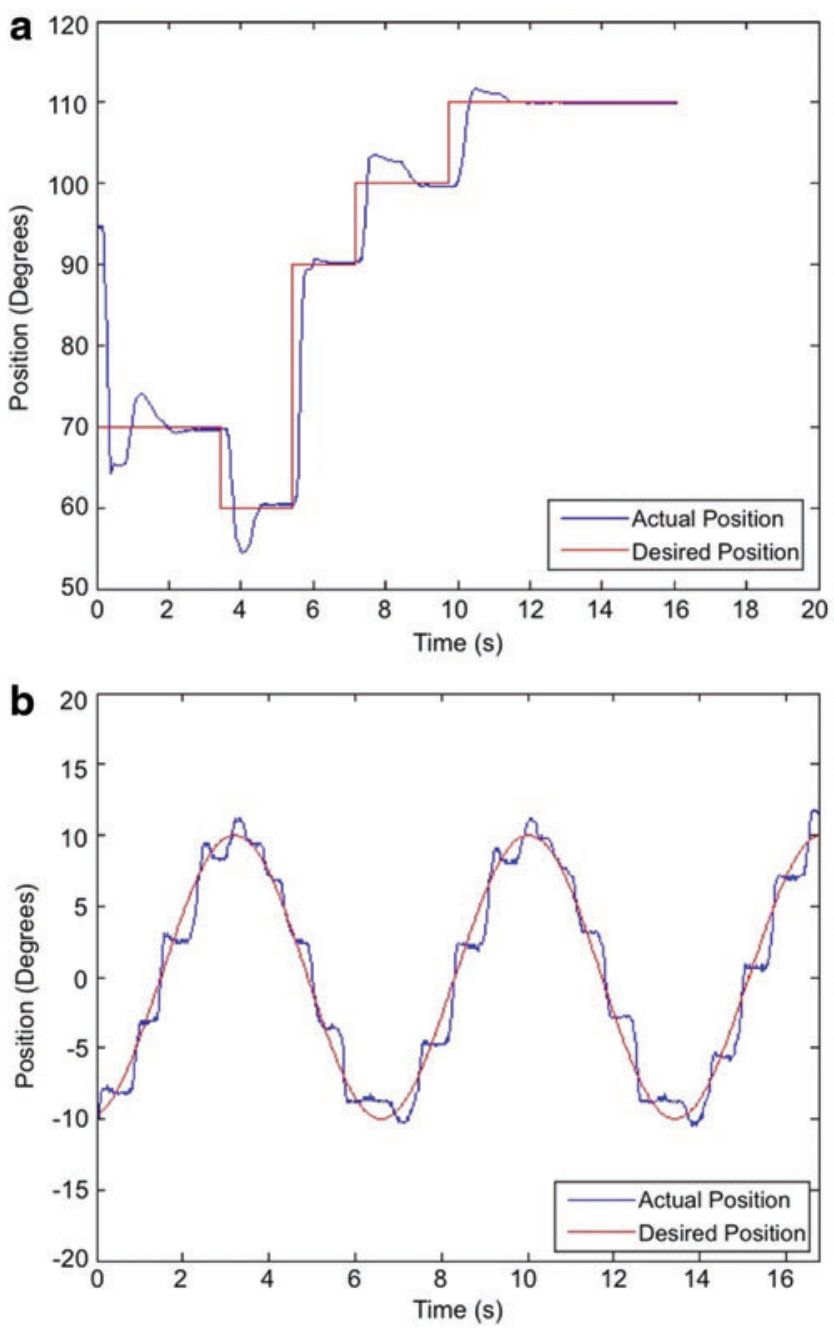

FIG. 12. Experimental results of PID-SM combined control showing fast overall response time, relatively high damping, and low steady-steady error. (a) Multistep response. (b) Sinusoidal response. Color images available online at www.liebertpub.com/soro

the PID or SM controllers alone and with reduced oscillations. Moreover, the pulsed SM reliably corrects steady-state error without hunting and often with little or no overshoot. Figure 12 shows the results of control experiments under no load using this third controller.

\section{Conclusion and Future Work}

In combining elements of rigid and soft robotics, as well as designing lightweight joints with low part counts, the robotic arm addresses many of the drawbacks of rigid and soft systems. Although heavier than purely soft systems, it is still lightweight for its size ( $117 \mathrm{~cm}$ long and weighing $1.2 \mathrm{~kg}$ ), mechanically compliant through the use of pneumatic air muscles and inflatable padding, and accurate by confining structural deformations to discrete joints, where they can be easily and reliably detected.

The arm still has a few drawbacks. In its current configuration, it has a relatively low maximum force output, the controllers exhibit pseudo-underdamped behaviors, and although the arm as a whole is compliant and the padding covers most of the internal structure, some rigid components remain exposed. These are all solvable with our current system, and will be addressed in future work. For example, with a more refined controller and by using more powerful air muscles (which are currently under development), the arm can be improved in terms of strength, speed, and accuracy. Adding another DOF to the arm would also improve its ability to perform human-like motions, such as pouring water from a bottle, and extending our control over passive tendons in the hand through the use of shape memory alloys would allow for variable elasticity. Once these improvements are implemented, the arm may possess all of the practical qualities necessary for a domestic environment.

Although the controllability of the arm was shown both in simulations and in experiments, it was based on a single joint control. To prove the overall efficacy and practicality of the proposed system, more thorough experiments need to be done with multijoint control for various positions in a system level. In addition, force control with dynamic motions should be investigated by equipping the arm with multiaxis forcetorque sensors in the future.

Another area of future work is control of inflatable sleeves with embedded soft sensors. The embedded sensors will not only be able to measure the level of air pressures but also detect external contacts. Then, actively controlling the air pressure of the sleeves depending on situations will be possible, which will make the robot more autonomous and interactive with environments.

\section{Acknowledgments}

This work was supported, in part, by National Aeronautics and Science Administration (NASA) under Small Business Innovation Research (SBIR) Contract NNX14CJ39P and, in part, by Technology Innovation Program (No. 2017-10069072) funded by the Ministry of Trade, Industry \& Energy (MOTIE), Korea, whose support is greatly appreciated.

\section{Author Disclosure Statement}

No competing financial interests exist.

\section{References}

1. Prassler E, Ritter A, Schaeffer C, Fiorini P. A short history of cleaning robots. Auton Rob 2000;9:211-226.

2. Burgard W, Cremers AD, Fox D, Hähnel D, Lakemeyer G, Schulz D, et al. Experiences with an interactive museum tour-guide robot. Artif Intell 1999;114:3-55.

3. Diallo AD, Gobee S, Durairajah V. Autonomous tour guide robot using embedded system control. Procedia Comput Sci 2015;76:126-133.

4. Intelligent Unmanned Ground Vehicles: Autonomous Navigation Research at Carnegie Mellon. Hebert MH, Thorpe CE, Stentz A. (Eds). New York, NY: Springer Science+Business Media, 1997.

5. Berger C, Rumpe B. Autonomous driving -5 years after the Urban Challenge: the anticipatory vehicle as a cyber-physical system. In: Proceedings IEEE/ACM International Conference on Automated Software Engineering (ASE2012), Essen, Germany, September 2012, pp. 789-798.

6. Bicchi A, Tonietti G. Fast and "soft-arm" tactics. IEEE Robot Autom Mag 2004;11:22-33. 
7. Khatib O. Real-time obstacle avoidance for manipulators and mobile robots. Int J Rob Res 1986;5:90-98.

8. De Santis A, Siciliano B. Safety issues for human-robot cooperation in manufacturing systems. In: Proceedings Tools and Perspectives in Virtual Manufacturing, Napoli, Italy, July 2008.

9. Park JJ, Kim HS, Song JB. Safe robot arm with safe joint mechanism using nonlinear spring system for collision safety. In: Proceedings IEEE International Conference Robotics and Automation (ICRA'09), Kobe, Japan, May 2009, pp. 3371-3376.

10. Yoon SS, Kang S, Yun SK, Kim SJ, Kim YH, Kim M. Safe arm design with MR-based passive compliant joints and viscoelastic covering for service robot applications. J Mech Sci Technol 2004;19:1835-1845.

11. Rus D, Tolley M. Design, fabrication and control of soft robots. Nature 2015;521:467-475.

12. Lipson H. Challenges and opportunities for design, simulation, and fabrication of soft robots. Soft Robotics 2012;1: 21-27.

13. Park YL, Chen B, Pérez-Arancibia B, Young D, Stirling L, Wood RJ, et al. Design and control of a bio-inspired soft wearable robotic device for ankle-foot rehabilitation. Bioinspir Biomim 2014;9:016007.

14. Park YL, Chen B, Majidi C, Nagpal R, Goldfield E. Active modular elastomer sleeve for soft wearable assistance robots. In: Proceedings IEEE/RSJ International Conference Intelligent Robots and Systems (IROS'12), Vilamoura, Portugal, September 2012, pp. 1595-1602.

15. Li T, Nakajima K, Calisti M, Laschi C, Pfeifer R. Octopus inspired sensorimotor control of a multi-arm soft robot. In: Proceedings IEEE International Conference Mechatronics and Automation, Chengdu, China, August 2012, pp. 948-955.

16. Sanan S, Moidel JB, Atkeson CG. Robots with inflatable links. In: Proceedings IEEE/RSJ International Conference on Intelligent Robots and Systems (IROS'09), St. Louis, MO, October 2009, pp. 4331-4336.

17. Sanan S, Ornstein MH, Atkeson CG. Physical human interaction for an inflatable manipulator. In: Proceedings IEEE International Conference on Engineering Medicine and Biology Society (EMBC'11), Boston, MA, August 2011, pp. 7401-7404.

18. Qi R, Lam TL, Xu Y. Kinematic modeling and control of a multi-joint soft inflatable robot arm with cable-driven mechanism. In: Proceedings IEEE International Conference on Robotics and Automation (ICRA'14), Hong Kong, China, May 2014, pp. 4819-4824.

19. Hunter IW, Hollerbach JM, Ballantyne J. A comparative analysis of actuator technologies for robotics. Hollerbach J, Craig JJ, Lozano-Perez T. (Eds). The Robotics Review 2. Cambridge, MA: MIT Press, 1992, pp. 299-342.

20. Tonietti G, Schiavi R, Bicchi A. Design and control of a variable stiffness actuator for safe and fast physical human/ robot interaction. In: Proceedings IEEE International Conference on Robotics and Automation (ICRA'05), Barcelona, Spain, April 2005, pp.523-531.

21. Tonietti G, Bicchi A. Adaptive simultaneous position and stiffness control for a soft robot arm. In: Proceedings IEEE/ RSJ International Conference on Intelligent Robots and Systems (IROS'02), Lausanne, Switzerland, October 2002, Vol. 2, pp. 1992-1997.

22. Albu-Schaffer A, Eiberger O, Grebenstein M, Haddadin S, Ott C, Wimbock T, et al. Soft robotics. IEEE Robot Autom Mag 2008;15:20-30.
23. Mahl T, Hildebrandt A, Sawodny O. A variable curvature continuum kinematics for kinematic control of the Bionic Handling Assistant. IEEE Trans Rob 2015;30:935-949.

24. Grezesiak A, Becker R, Verl A. The Bionic Handling Assistant: a success story of additive manufacturing. Assembly Autom 2011;31:329-333.

25. Wilson M. Festo drives automation forwards. Assembly Autom 2011;31:12-16.

26. Sanan S, Lynn PS, Griffith T. Pneumatic torsional actuators for inflatable robots. J Mech Rob 2014;6:031003.

27. Pratt GA, Williamson MM. Series elastic actuators. In: Proceedings IEEE/RSJ International Conference on Intelligent Robots and Systems (IROS'95), Pittsburgh, PA, August 1995, pp. 399-406.

28. Zinn M, Roth B, Khatib O, Salisbury JK. New actuation approach for human-friendly robot design. Int J Rob Res 2004;23:379-398.

29. Shin D, Sardellitti I, Park YL, Khatib O, Cutkosky MR. Design and control of a bio-inspired human-friendly robot. Int J Rob Res 2010;29:571-584.

30. Mori M. The uncanny valley. Energy 1970;7:33-35.

31. Duffy BR. Anthropomorphism and the social robot. Rob Auton Syst 2003;42:177-190.

32. Walters ML, Syrdal DS, Dautenhahn K, Boikhorst R, Koay KL. Avoiding the uncanny valley: robot appearance, personality and consistency of behavior in an attention-seeking home scenario for a robot companion. Auton Rob 2008;24:159-178.

33. Chou CP, Hannaford B. Measurement and modeling of McKibben pneumatic artificial muscles. IEEE Trans Rob Autom 1996;12:90-102.

34. Tondu B. Lopez P. Modeling and control of McKibben artificial muscle robot actuators. IEEE Control Syst Mag 2000;20:15-38.

35. Daerden F, Lefeber D. The concept and design of pleated pneumatic artificial muscles. Int J Fluid Power 2001;2: 41-50.

36. Park YL, Santos J, Galloway K, Goldfield EC, Wood RJ. A soft wearable robotic device for active knee motions using flat pneumatic artificial muscles. In: Proceedings IEEE International Conference on Robotics and Automation (ICRA'14), Hong Kong, China, May 2014, pp. 4805-4810.

37. Wehner M, Quindan B, Aubin P, Martinez-Villalpando E, Baumann M, Stirling L, et al. A lightweight soft exosuit for gait assistance. In: Proceeding IEEE International Conference on Robotics and Automation (ICRA'13), Karlsruhe, Germany, May 2013, pp. 3347-3354.

38. Jiang L, Low K, Costa J, Black RJ, Park YL. Fiber optically sensorized multi-fingered robotic hand. In: Proceeding IEEE/RSJ International Conference on Intelligent Robots and Systems (IROS'15), Hamburg, Germany, September 2015, pp. 1763-1768.

39. Patre PM, MacKunis W, Makkar C, Dixon WE. Asymptotic tracking for systems with structured and unstructured uncertainties. IEEE Trans Control Syst Technol 2008;16:373-379.

Address correspondence to:

Yong-Lae Park

Department of Mechanical and Aerospace Engineering Seoul National University

1 Gwanak-ro, Gwanak-gu Seoul, 08826

Korea

E-mail: ylpark@snu.ac.kr 